\title{
3D Spectral reverse time migration with no-wraparound absorbing conditions
}

\author{
Ernesto Bonomi, Leesa Brieger, Carlo Nardone, and Enrico Pieroni, Geophysics Area, CRS 4
}

\section{Summary}

Comparative studies of methods of reverse time migration (RTM) show that spectral methods for calculating the Laplacian impose the least stringent demands on discretization stepsize; thus with spectral methods, the grid refinements often required by other methods can be avoided. Implemented with absorbing boundary conditions, which are energy-tuned to give good absorption at the boundaries, these spectral methods can be used effectively for migration, without suffering the problems of wraparound which have traditionally plagued them (Furumyra and Takenaka, 1995).

\section{Introduction}

Reverse time migration for poststack imaging is based on the full 3D d'Alembert wave equation. Other imaging techniques based on the one-way equation, such as depth extrapolation, can only propagate partial information and so cannot achieve the resolution of RTM. For this reason, RTM poststack migration can produce a subsurface reconstruction superior to that of most other poststack migration methods, as has been observed in industrial applications.

The wave equation can be formulated in Hamiltonian form as

$$
\begin{aligned}
\dot{P}(x, y, z, t) & =Q(x, y, z, t), \\
\dot{Q}(x, y, z, t) & =c^{2} \nabla^{2} P(x, y, z, t),
\end{aligned}
$$

for $t \in[0, T]$ and $(x, y, z) \in \Omega$. Time-marching proceeds backward from final acquisition time $T$, with homogeneous final conditions on $P$ and $Q$, up to time zero, where the imaging principle describes the subsurface reflectivity. At each time step the stack is imposed as the surface boundary condition. The finite volume $\Omega$, necessary because the numerical solution requires a finite computational bulk and not because $\partial \Omega$ represents a physical barrier, requires boundary conditions that mimic a transparent boundary. Typically some implementation of absorbing boundary condition is used to force waves to flow out of $\Omega$.

\section{Spectral RTM: stability and accuracy}

The most important aspect of our numerical scheme for solving the Hamiltonian system, Eqn.(1), is the use of Fourier space for evaluating the Laplacian - using forward and inverse discrete Fourier transforms $(\mathcal{D F} \mathcal{T})$ (Briggs and Henson, 1995):

$$
\nabla^{2} P^{(n)}=\mathcal{D} \mathcal{F} \mathcal{T}^{-1}\left[-\|\vec{k}\|^{2} \mathcal{D} \mathcal{F} \mathcal{T}\left(P^{(n)}\right)\right]
$$

where $\vec{k}=\left(k_{x}, k_{y}, k_{z}\right)^{T}$.

The system is then time-integrated using the following leap-frog scheme (written without showing the spatial dependencies):

$$
\left\{\begin{array}{l}
Q^{\left(n+\frac{1}{3}\right)}=Q^{(n)}+\frac{1}{6} \Delta t c^{2} \nabla^{2} P^{(n)} \\
P^{\left(n+\frac{1}{2}\right)}=P^{(n)}+\frac{1}{2} \Delta t Q^{\left(n+\frac{1}{3}\right)} \\
Q^{\left(n+\frac{2}{3}\right)}=Q^{\left(n+\frac{1}{3}\right)}+\frac{2}{3} \Delta t c^{2} \nabla^{2} P^{\left(n+\frac{1}{2}\right)} \\
P^{(n+1)}=P^{\left(n+\frac{1}{2}\right)}+\frac{1}{2} \Delta t Q^{\left(n+\frac{2}{3}\right)} \\
Q^{(n+1)}=Q^{\left(n+\frac{2}{3}\right)}+\frac{1}{6} \Delta t c^{2} \nabla^{2} P^{(n+1)}
\end{array}\right.
$$

Equations (3) permit an improvement in accuracy to fourth order without any increase in memory requirements over the central finite difference method, and preserve the time-reversibility of the wave equation (Sexton and Weingarten, 1992), (R. D. Skeel and Schlick, 1997). This scheme also gives direct access to the field time derivative $Q$ (the generalized momentum), which will be useful for implementing absorbing boundary conditions.

Standard Von Neumann analysis yields the following stability condition:

$$
c \Delta t \sqrt{\frac{1}{\Delta x^{2}}+\frac{1}{\Delta y^{2}}+\frac{1}{\Delta z^{2}}} \leq \frac{\sqrt{6}}{\pi} .
$$

Further analysis shows that the stability condition for the leap-frog scheme allows a time step 1.22 times larger than that for central differences for the time integration.

Eliminating $Q$ from Equations (3), the leap-frog scheme reduces to

$$
\frac{P^{n+1}-2 P^{n}+P^{n-1}}{\Delta t^{2}}=c^{2} \nabla^{2} P^{n}+\frac{c^{4} \Delta t^{2}}{18} \nabla^{4} P^{n},
$$

and using Fourier transforms to write the overall method in the $(\omega, \vec{k})$-domain, we derive the discrete dispersion relation

$$
4 \sin ^{2} \frac{\omega \Delta t}{2}=\kappa^{2}-\frac{1}{18} \kappa^{4}, \quad \kappa=c \Delta t\|\vec{k}\|,
$$

which determines whether different wavelengths propagate at different speeds. In order to maintain accuracy and mimic the dispersion characteristics of the actual solution, a numerical scheme should give rise to a dispersion relation which is "not far" from the continuous one, $\omega_{c}^{2} \Delta t^{2}=\kappa^{2}$. Fig.1(a) plots the dimensionless frequency given by the discrete and by the continuous dispersion relations for $\omega \geq 0$. We thus impose a dispersion condition requiring that the relative difference between the continuous and discrete dispersion relations be bounded by a tolerance $\epsilon$ :

$$
E(\kappa)=\frac{\omega \Delta t-\kappa}{\kappa} \leq \epsilon
$$




\section{Spectral RTM with absorbing boundaries}

where $\omega \Delta t$ is defined implicitly by the discrete dispersion relation (6) and where, due to symmetry around 0 , we consider only positive values of $\omega$, for which $E(\kappa)$ is then positive, see Fig.1(b). For a given $\epsilon, \kappa_{\epsilon}$ represents the value of the dimensionless wavenumber for which $E\left(\kappa_{\epsilon}\right)=$ $\epsilon$; the corresponding dimensionless frequency is $(\omega \Delta t)_{\epsilon}=$ $\kappa_{\epsilon}(1+\epsilon)$. Due to the monotonicity of $E(\kappa)$, see Fig.1(b), $E(\kappa)<\epsilon$ whenever $0<\kappa<\kappa_{\epsilon}$, and $(\omega \Delta t)_{\epsilon}$ is the largest dimensionless frequency for which the dispersion relation error is bounded by $\epsilon$. Since $\omega<2 \pi f_{\max }$, where $f_{\max }$ represents the largest frequency in the band of the seismic section, we can deduce a limit on $\Delta t$ that guarantees the dispersion relation tolerance. As long as

$$
\Delta t \leq \frac{\kappa_{\epsilon}(1+\epsilon)}{2 \pi f_{\max }},
$$

then $\omega \Delta t<\kappa_{\epsilon}(1+\epsilon)$ and the dispersion relation error is to within the desired tolerance for all frequencies present in the seismic section.

For $\epsilon=1 \%$, we find $\kappa_{\epsilon}=0.83633$ for the leap-frog scheme and $\kappa_{\epsilon}=0.48336$ for central finite differences; thus the dispersion condition for the leap-frog scheme allows a time step about twice as large as that for central differences.

It is significant that the dispersion analysis leads to a condition on stepsize $\Delta t$ alone. This has been fundamental to our choice of spectral methods in the space variables. For other, non-spectral methods in space, the presence of $\Delta x, \Delta y$, and $\Delta z$ in the dispersion relations can lead to severe restrictions on these stepsizes when a given tolerance is imposed on the dispersion relation error. Such restrictions can necessitate significant refinement of the space grids, increasing by many times the computational size of the problem. Spectral methods in space can furnish a dispersion relation tolerance with no spatial refinement ever required.

Both the stability and dispersion conditions must be satisfied. With $\Delta x, \Delta y$, and $\Delta z$ fixed by the acquisition grid and the desired resolution in $z$, the stability condition (4) is used to define a bound on $\Delta t$. The minimum between this and the bound from the dispersion condition (8) is finally imposed on $\Delta t$. With the spatial discretization and frequencies typical of seismic applications (for instance, $\Delta x=\Delta y=25 m, \Delta z=10 m, f_{\max }=70 \mathrm{~Hz}$ ), it is often the dispersion condition which is the more stringent.

\section{Energy-tuned absorbing boundary conditions}

From the wave equation (1), we can derive the continuity equation

$$
\frac{d H}{d t}=\int_{\partial \Omega} \vec{J} d \vec{\sigma}, \quad \vec{J}=Q \vec{\nabla} P
$$

where $H(t)$ is interpreted as the energy inside $\Omega$ at time $t$,

$$
H(t)=\frac{1}{2} \int_{\Omega}\left[(\overrightarrow{\nabla P})^{2}+(Q / c)^{2}\right] d V,
$$

and $\vec{J}$ is the local energy flux density. If $\vec{J} \cdot d \vec{\sigma}$ vanishes everywhere on the boundary $\partial \Omega$, then $d H / d t=0$ and en- ergy is conserved. Otherwise, wherever $\vec{J}$ points intward, energy increases locally and wherever $\vec{J}$ points outward, energy decreases locally (Broeze and Daalen, 1992). From these observations follows a necessary energy condition for absorption at a boundary: $d H / d t \geq 0$.

A well-established way of imposing this condition and implementing an absorbing boundary is based on the observation that an impulse traveling along direction $\hat{n}$ with velocity $c$ satisfies the simplest paraxial wave equation

$$
\partial_{t} P-c \hat{n} \cdot \vec{\nabla} P=0
$$

Imposing equation (11) on $\partial \Omega$ and identifying $\hat{n}$ as the normal to the boundary, we see that $d H / d t \geq 0$ is indeed true. This does not, however, prevent the existence of reflected waves corresponding to other (non-normal) wave angles.

Our strategy (Bonomi and Pieroni, 1998) for constraining all waves to be absorbed by the boundary is based on imposing $\vec{J} \cdot \hat{n} \leq 0$ by locally reversing the sign of $Q$ on the boundary wherever the projection of $\vec{J}$ along the normal $\hat{n}$ is negative:

$$
\begin{gathered}
\text { FOR }(\mathrm{x}, \mathrm{y}, \mathrm{z}) \in \partial \Omega \text { WHERE } \overrightarrow{\mathrm{J}} \cdot \hat{\mathrm{n}}<0 \\
\operatorname{DO} \mathrm{Q}(\mathrm{x}, \mathrm{y}, \mathrm{z}, \mathrm{t}) \leftarrow-\mathrm{Q}(\mathrm{x}, \mathrm{y}, \mathrm{z}, \mathrm{t}) .
\end{gathered}
$$

Remark that while the energy evolution is altered by reversing the sign of $Q$, the instantaneous value of the energy is not, and conservation of energy is intact. In contrast to equation (11) alone, this mechanism also preserves the local energy flux strength $\|\vec{J}\|$. Reversing the sign of $Q$, the main ingredient of the absorbing boundary that we propose, can be easily applied using the Hamiltonian formulation of wave propagation because the generalized momentum $Q$ is treated as an independent field.

In the actual implementation, we consider a bounded domain $\Sigma$ which contains $\Omega$. The absorbing condition is now rendered effective by extending the $Q$ reversal to all points of $\Sigma \backslash \Omega$. To evaluate $\vec{\nabla} P$, we compute first derivatives of $P$ using second order central finite differences. Numerical experiments confirm that energy is then trapped in a strip pattern inside this layer. To dissipate this unwanted energy, which is a source of numerical error, we implement the simplest absorbing boundary, equation (11), discretized with finite differences, on $\partial \Sigma$.

To summarize, a single time integration step consists of the following three phases: integrating the Hamiltonian equations in $\Sigma$, imposing mechanism (12) in $\Sigma \backslash \Omega$, and damping the residual energy by integrating the paraxial equation (11) on $\partial \Sigma$. Because the algorithm constrains energy not to increase in reverse time, the solution is guaranteed to be bounded by the initial data, thus ensuring well-posedness of the approach (Ha-Duong and Joly, 1994). 


\section{Spectral RTM with absorbing boundaries}

\section{Results}

With absorbing boundary conditions enhanced by Qreversal, the spectral approach gives good imaging results, that is, minimal reflection and no-wraparound, when the first derivatives, necessary for the $Q$-reversal, are evaluated by second order central finite differences. In fact, we tested also the evaluation of the first derivative by $\mathcal{D} \mathcal{F} \mathcal{T}$, or by costly implicit finite differences. In the first case we observed that reflection was minimized but the wraparound still was persistent, while in the second case we have not noticed any significant improvement with respect to the fastest central finite differences.

Fig.2 illustrates the effect of our $Q$-reversal mechanism on the migration of a synthetic data set in the case of a constant velocity field, $c=1500 \mathrm{~m} / \mathrm{s}$. The seismic section is a classical Ricker wavelet in time with time half width of $25 \mathrm{~ms}$ and a Gaussian in space with space half width of $68 \mathrm{~m}$; time sampling is $\Delta t=5 \mathrm{~ms}$. The spatial mesh has $N_{x}=128, N_{y}=64$ and $N_{z}=512$ equally spaced points: $\Delta x=\Delta y=\Delta z=17 \mathrm{~m}$. Shown are four snapshots of the evolving field, for $y$ fixed, taken at timesteps $160,400,680$ and 1120 for two examples - with and without $Q$-reversal. The paraxial equation, a component of the absorbing boundary, is present in both examples to dissipate energy.

In Fig.2(a) $Q$-reversal is applied in a layer 8 mesh points $(138 \mathrm{~m})$ thick, trapping energy that would otherwise be incorrectly reflected back into the domain. In Fig.2(b) $Q$-reversal is not applied and absorption is obtained by the paraxial equation alone. The Ricker wavelet is placed off-center to facilitate distinguishing between the reflected signal, which is of opposite phase with the incident wave, and wraparound, which is in phase. As the signal proceeds, the wave incidence becomes more parallel to the lateral boundary, and the paraxial equation becomes less efficient at absorbing outgoing energy. This effect is clear in Fig.2(b) with the appearance of reflections. In addition, the poor absorption at the boundary, coupled with the intrinsic periodicity of the $\mathcal{D} \mathcal{F} \mathcal{T}$, causes misleading wraparound, also clear in Fig.2(b). On the other hand, with the $Q$-reversal mechanism, Fig.2(a), the re-entry of unabsorbed energy from the boundary is inhibited, cutting down on both reflections and wraparound.

\section{Conclusions}

With the spectral approach to RTM, the dispersion condition for accuracy requires no further spatial refinement; every other method does. The subsequent economy of problem size for spectral RTM with no-wraparound boundary conditions makes feasible industrial poststack migration by solving the full wave equation. RTM, the most accurate poststack migration method, should be considered a viable compromise between the cheaper oneway poststack migration and all-out prestack migration.

\section{Acknowledgements}

The authors wish to thank M. Teresa Arienti, Luca Cazzola, Paolo Marchetti and Claudio Zenucchini for stimulating discussions as well as for many tests of the algorithm - carried out in the framework of the collaboration between CRS4 and ENI-AGIP, E\&P Division.

\section{References}

Bonomi, E., and Pieroni, E., 1998, Energy-tuned absorbing boundary conditions Proc. fourth SIAM Int. Conf. on Mathematical and Numerical Aspects of Wave Propagation, June 1-5, 1998, Colorado School of Mines, Golden, Colorado., SIAM.

Briggs, W. L., and Henson, V. E., 1995, The DFT: SIAM, Philadelphia, PA.

Broeze, J., and Daalen, E. F. G. V., 1992, Radiation boundary conditions for the two-dimensional wave equation from a variational principle: Math. Comp., $\mathbf{5 8}, 55-71$.

Furumyra, T., and Takenaka, H., 1995, A wraparaound elimination technique for the pseudospectral wave synthesis using an antiperiodic extension of the wavefield: Geophysics, 60, 302-307.

Ha-Duong, T., and Joly, P., 1994, On the stability analysis of boundary conditions for the wave equation by energy methods: the homogeneous case: Math. Comp., 67, 539-563.

R. D. Skeel, G. Z., and Schlick, T., 1997, A family of symplectic integrators: stability, accuracy and molecular dynamics applications: SIAM J. Sci. Comput., 18, 203-222.

Sexton, J. C., and Weingarten, D. H., Hamiltonian evolution for the hybrid monte carlo algorithm:, Technical Report RC 177668, IBM Research Division, T. J. Watson Research Center, Yorktown Heights, NY 10598, February 1992. 
Spectral RTM with absorbing boundaries
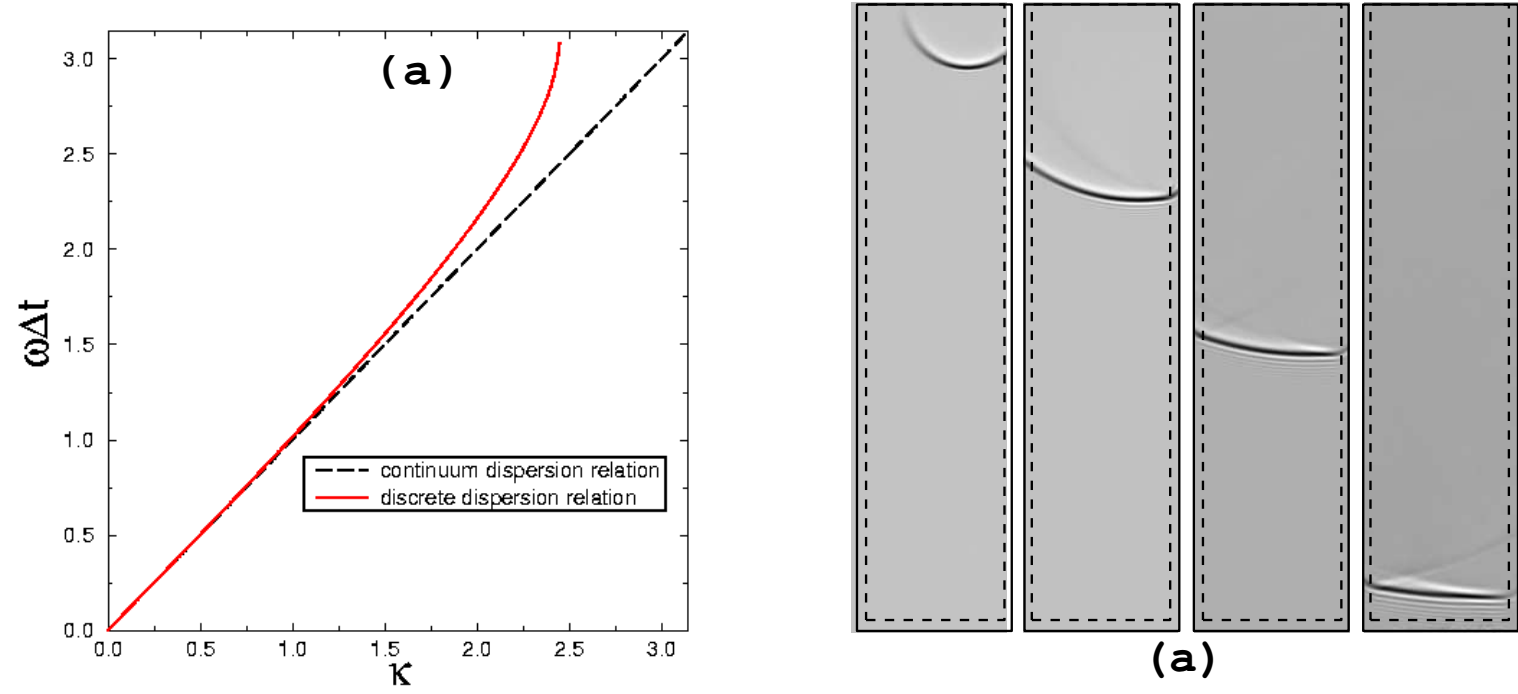

(a)
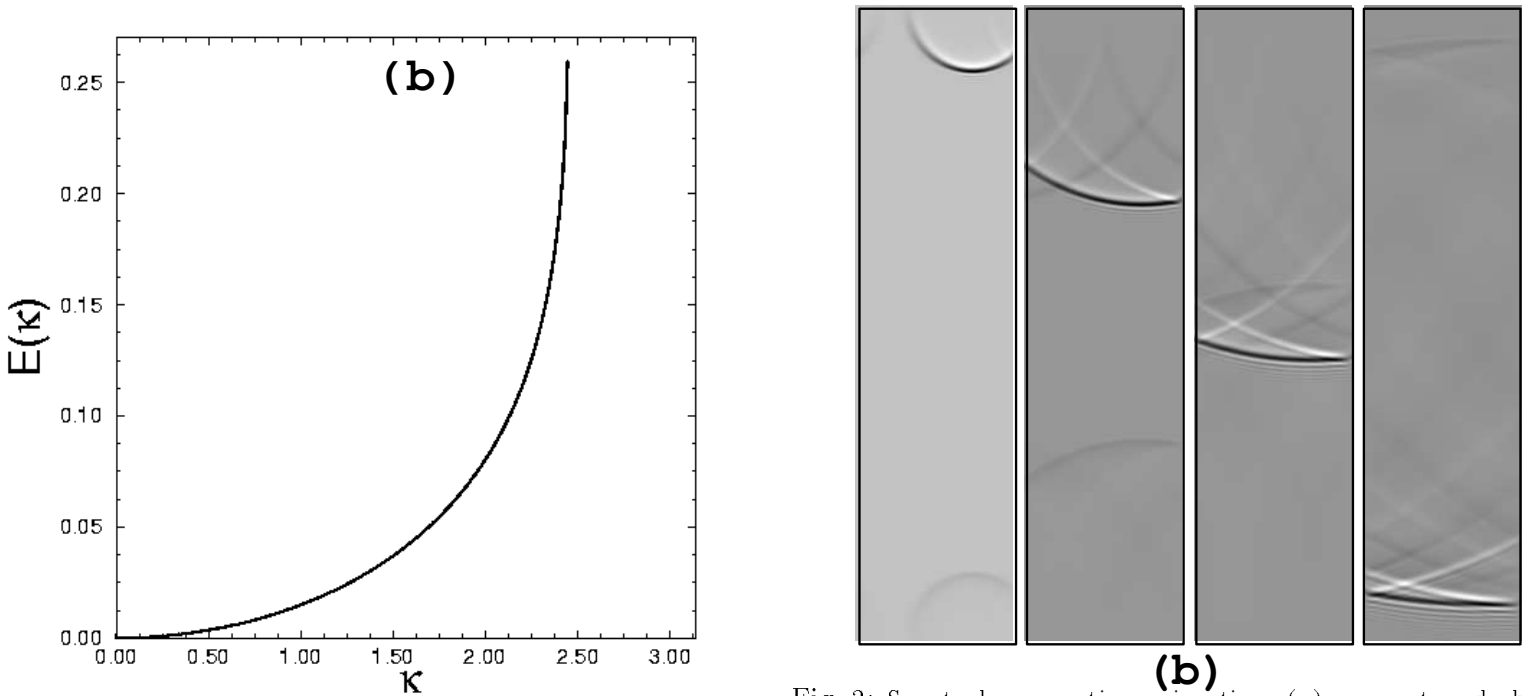

(b)

Fig. 1: (a) Discrete and continuum dispersion relations for $\omega \geq 0$; (b) Relative difference between the continuous and discrete dispersion relations for $\omega>0$.

Fig. 2: Spectral reverse time migration: (a) energy-tuned absorbing boundary conditions, dashed lines delimit the $Q$-reversal layer; (b) absorption at the boundaries imposed by the paraxial equation (11) alone - no $Q$-reversal. 